\title{
ANÁLISE DE AGRUPAMENTOS ASSOCIADA A DIFRATOMETRIA DE RAIOS-X: UMA CLASSIFICAÇÃO MINERALÓGICA PRÁTICA DE BAUXITAS E SEUS PRODUTOS DE DIGESTÃO BAYER
}

\author{
C. C. A. MELO ${ }^{1, *}$, K. S. OLIVEIRA ${ }^{2}$, R. S. ANGÉLICA ${ }^{2}$, S. P. A. PAZ $Z^{1,2}$ \\ ${ }^{1}$ Universidade Federal do Pará, Inst. de Tecnologia, Prog. de Pós-Grad. em Engenharia de Recursos Naturais da \\ Amazônia, ${ }^{2}$ Universidade Federal do Pará, Inst. de Geociências, Prog. de Pós-Grad. em Geologia e Geoquímica \\ eng.caiomelo@hotmail.com*
}

Submetido 10/10/2017 - Aceito 27/11/2017

DOI: $10.15628 /$ holos.2017.6387

\section{RESUMO}

Este trabalho teve como objetivo principal utilizar a ferramenta de análise de agrupamentos associada a difratometria de raios- $X$ para classificar mineralogicamente horizontes bauxíticos e seus sólidos residuais da digestão Bayer. Foram utilizadas 172 amostras: 168 de horizontes bauxíticos e 4 de finos cauliníticos derivados da lavagem de bauxitas destes horizontes, da Mina Miltônia 3, Paragominas-PA. Os resultados mostram que as fases mineralógicas presentes em todas as amostras de bauxitas foram: gibbsita, caulinita, Al-goethita, hematita e anatásio. Industrialmente, a distinção desses horizontes é feita quimicamente (teor de alumina aproveitável e sílica reativa - medida indireta da quantidade de gibbsita e caulinita, respectivamente). No entanto, através da análise de agrupamentos, tem-se mais um parâmetro para distinção das fases, no caso a "cristalinidade", apurando a classificação mineralógica/cristaloquímica dos diferentes tipos de bauxitas. Para os sólidos residuais da digestão foi possível observar as fases inertes do processo: Al-goethita, hematita e anatásio; ausência e/ou presença de sodalita, caulinita e gibbsita. Também foi possível pela análise de agrupamentos diferenciar as litologias que continham caulinita(s) mais reativa(s) na digestão Bayer.

PALAVRAS-CHAVE: DRX, Análise de Agrupamentos, Bauxita, Sílica Reativa.

\section{Cluster Analysis from X-ray Diffraction data: A practical tool for mineralogical classification of bauxites and related products from the Bayer digestion}

\begin{abstract}
The main objective of this work is to use the Cluster Analysis statistical tool from X-ray diffraction data to classify different bauxite types and their related products from the Bayer Digestion. 172 samples were used (168 bauxites and 4 samples from kaolinite-rich fines from bauxite washing. All samples come from the Miltônia 3 mine, Paragominas-PA. The results show that gibbsite, kaolinite, Al-goethite, hematite and anatase are the main minerals present in all samples. In the industry, quality control is carried out mainly using wet chemical methods (available alumina and reactive silica, an
\end{abstract}

indirect measure of the amount of gibbsite and kaolinite, respectively). Meanwhile, by using Cluster Analysis of XRD data, an additional parameter can be evaluated, the "crystallinity" of the minerals from the samples. For the residual solids of the digestion it was also possible to observe the following inert phases: Algoethite, hematite and anatase; besides the presence or absence of sodalite, kaolinite and gibbsite. It was also possible to observe and differentiate lithologies with kaolinites more reactive in the Bayer digestion. 


\section{INTRODUÇÃO}

O Brasil possui a terceira maior reserva de bauxita do mundo, sendo que boa parte está concentrada nos depósitos da região amazônica (Paragominas, Juruti, Trombetas e Almerim) (Peixoto e Horbe, 2008). A Província bauxitífera de Paragominas ocupa uma área da ordem de $50.000 \mathrm{~km}^{2}$ e está localizada na região Leste do Pará e Oeste do Maranhão (Kotschoubey et al., 2005).

A bauxita é um material regolítico, produto do intemperismo de rochas aluminossilicatadas com elevada concentração de minerais de alumínio que perfaz reservas economicamente exploráveis. Nos depósitos bauxíticos brasileiros o mineral de interesse mais comum é a gibbsita e, de maneira geral, associada a caulinita, quartzo, hematita, goethita e anatásio (Freyssinet et al., 2005). Na indústria do alumínio metálico, o interesse econômico do minério depende principalmente das quantidades de gibbsita e caulinita, que são os principais minerais de interesse e de ganga, respectivamente. Por isso, é tão fundamental o controle mineralógico.

Tradicionalmente, tem-se desde a prospecção do minério até a lavra um controle químico ao invés de mineralógico, são os parâmetros de composição química: $\mathrm{Fe}_{2} \mathrm{O}_{3}, \mathrm{Al}_{2} \mathrm{O}_{3}, \mathrm{SiO}_{2}, \mathrm{TiO}_{2}$ e Perda ao Fogo, além de $\mathrm{AvAl}_{2} \mathrm{O}_{3}$ (Alumina Aproveitável) e $\mathrm{RxSiO}_{2}$ (Sílica Reativa). Esses dois últimos são os parâmetros de controle fundamentais em todo o processo da mineração de bauxita metalúrgica, são reconhecidamente de análise química via úmida trabalhosa e de alta incerteza para $\mathrm{RxSiO}_{2}$ ( $\mathrm{Paz}$ et al., 2017a). Mas são métodos consolidados ao invés dos métodos mineralógicos.

Devido as conhecidas dificuldades vividas no reconhecimento dos horizontes lateríticosbauxíticos durante o mapeamento das frentes de lavra, pergunta-se se a difratometria de raios- $X$ não traria mais praticidade nesse ponto de demarcação e tomada de decisão, uma vez que se tem informações diretas das formas estruturais que revelam a história de cada fase mineral. Pela análise de agrupamentos, por exemplo, a comparação baseada na intensidade e posição dos picos difratados equivale a comparação entre as principais fases minerais das amostras.

A técnica de agrupamento hierárquico agrupa amostras por suas semelhanças cristalográficas e composicionais, usando análise estatística por componentes principais. De forma muito simples e rápida, a técnica pode ser aplicada sob as medidas de difração de raios-X (difratogramas) sem nenhum tratamento prévio ou conhecimento da mineralogia das amostras. Por isso, a análise de agrupamentos tem se tornado uma ferramenta útil e ágil na classificação de minérios, principalmente quando se tem uma variabilidade muito grande no mesmo depósito. A técnica vem sendo utilizada principalmente com o objetivo de se avaliar, de forma mais prática, os resultados de análise química (Macchiarola et al., 2007, Shimizu et al., 2012).

O presente trabalho consistiu na classificação de horizontes bauxíticos através da análise de agrupamentos, de acordo com as respectivas características cristaloquímicas e comparação dos resultados com os dados de análise química realizados pela empresa Hydro Mineração Paragominas. 
O trabalho ainda teve como objetivo avaliar os grupos (clusters) com relação ao grau de ordenamento estrutural das caulinitas presentes em cada horizonte bauxítico e com os produtos sólidos obtidos da digestão alcalina de amostras desses horizontes.

\section{MATERIAIS E MÉTODOS}

O presente estudo foi dividido em duas partes: (1) análise e classificação mineralógica de horizontes bauxíticos da mina Miltônia 3, Paragominas-PA; (2) análise e classificação mineralógica dos resíduos sólidos do processo de digestão Bayer em condições de laboratório a partir de finos cauliníticas do beneficiamento de bauxitas de um poço de pesquisa também da mina Miltônia 3, Paragominas-PA.

\subsection{Amostras Bauxíticas}

As amostras de bauxita utilizadas na primeira parte desse trabalho foram amostradas a partir de uma malha de sondagem contendo 23 furos espaçados de $25 \mathrm{~m} \times 25 \mathrm{~m}$, totalizando 168 amostras que compreendem os horizontes industrialmente classificados como: BN (bauxita nodular), BNC (bauxita nodular cristalizada), LF (laterita ferruginosa), BC (bauxita cristalizada) e BCBA (bauxita cristalizada bauxita amorfa), termos definidos em Silva et al. (2008). Os procedimentos de amostragem, diminuição de tamanho de partículas (cominuição), homogeneização (tamanho de partículas < 0,15 mm) e quarteamento das amostras foram realizados pela empresa Hydro Mineração Paragominas, a qual forneceu uma alíquota de $\sim 10 \mathrm{~g}$ de cada para a realização desse trabalho. Os dados de $\mathrm{AvAl}_{2} \mathrm{O}_{3}$ e $\mathrm{RxSiO}_{2}$ determinados no laboratório químico da empresa também foram fornecidos.

As amostras utilizadas na segunda parte do trabalho foram obtidas da lavagem de $\sim 12 \mathrm{~kg}$ de quatro horizontes bauxíticos (BN, BNC, BC e BCBA) de um poço de pesquisa (Coord. N/S 9637707 e Coord. E/W 0202041). A lavagem foi realizada em equipamento Hydro-Clean (Marca Haver \& Boecker, modelo de laboratório) seguida de classificação a 0,037 mm em peneirador vibratório (Marca Abronzinox, com peneiras quadradas de $50 \times 50 \mathrm{~cm}$ ) e deslamagem da fração passante em 0,037 $\mathrm{mm}$ em hidrociclone Krebbs de $25 \mathrm{~mm}$ de diâmetro operando a 25 psi com corte $\sim 10 \mu \mathrm{m}$. As frações overflow dessa etapa correspondem as amostras desse trabalho.

\subsection{Digestão alcalina}

O processo de digestão Bayer foi realizado em bombas Parr de aço inoxidável revestidas internamente de teflon, utilizando $1 \mathrm{~g}$ de ganga caulinítica e $25 \mathrm{~mL}$ de solução $\mathrm{NaOH} 5,0 \mathrm{M}$, variando o tempo de reação em 60,120, 180, 420 min e 3 dias. Todas as digestões foram conduzidas à $150^{\circ} \mathrm{C}$ e pressão autógena. Os resíduos sólidos insolúveis do processo de digestão Bayer foram lavados até $\mathrm{pH} \sim 7$ e secos em estufa à $105^{\circ} \mathrm{C}$ por $6 \mathrm{~h}$.

\subsection{Condições instrumentais para as medidas de Difratometria de Raios-X (DRX)}

As medidas foram realizadas em um difratômetro de raios- $X$ modelo X'PERT PRO MPD

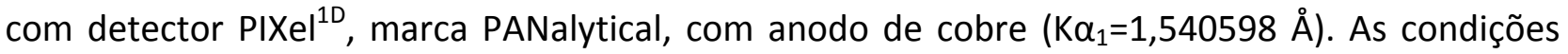
instrumentais utilizadas para as amostras de bauxita foram: varredura de 8 a $80^{\circ}$ em $2 \theta ; 40 \mathrm{kV} / 30$ $\mathrm{mA}$; tamanho do passo de $0,033^{\circ}$ em $2 \theta$ e $40 \mathrm{~s}$ o tempo/passo; para as gangas cauliníticas: 5 a 
$100^{\circ}$ em $2 \theta, 40 \mathrm{kV} / 40 \mathrm{~mA}$, tamanho do passo de $0,02^{\circ}$ em $2 \theta$ e $90 \mathrm{~s}$ o tempo/passo. 0 software $X^{\prime}$ Pert High Score versão 3.0, da PANalytical, foi usado na identificação de fases cristalinas e nas Análises de Agrupamentos.

Os parâmetros de comparação adotados nas análises de agrupamentos (cluster analysis) foram: "perfil + picos" como fonte de dados, "posição + intensidade" como tipo de comparação com threshold de 75\%; e os parâmetros de agrupamentos foram: medida das distâncias "Euclidiana", distância média "average linkage" como método de distância entre os clusters, e Cut-off manual.

\section{RESULTADOS E DISCUSSÃO}

\subsection{Caracterização mineralógica}

Conforme observado na Figura 1 todas as amostras dos horizontes bauxíticos e gangas cauliníticas apresentam padrões difratométricos semelhantes quanto as fases minerais: caulinita $\left(d_{001}=7,14 \AA\right.$ e $\left.d_{002}=3,57 \AA\right)$, gibbsita $\left(d_{002}=4,84 \AA\right.$ ), Al-goethita (com variação do pico referente ao plano $\mathrm{d}_{101}=4,12-4,16 \AA$ devido a variação de $\mathrm{Al}$ na solução sólida de até $33 \%$ substituindo o Fe) (Thiel, 1963; Paz et al., 2017b), anatásio ( $\left.d_{011}=3,52 \AA ̊ \AA\right)$ e hematita $\left(d_{104}=2,69 \AA ̊\right)$.

(a)

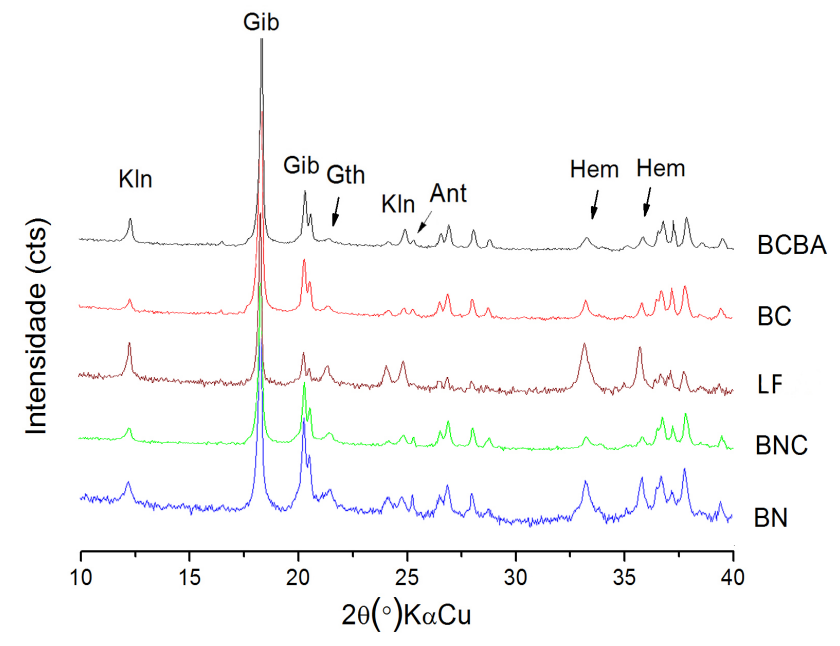

(b)

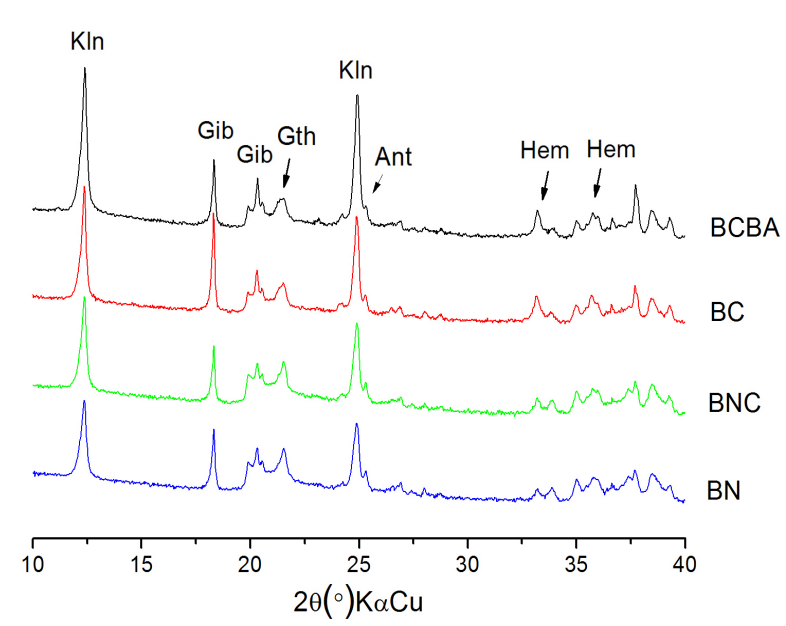

Legenda: KIn: Caulinita; Gib: Gibbsita; Gth: Goethita; Ant: Anatásio; Hem: Hematita.

Figura 1: Difratogramas de raios-X: (a) horizontes bauxíticos e (b) gangas cauliníticas.

\subsection{Classificação dos horizontes bauxíticos}

De acordo com as condições de agrupamento adotadas nesse trabalho, as amostras foram discriminadas em 8 grupos (clusters) e apenas uma amostra outlier não foi agrupada. Na Tabela 1 estão sumarizados as quantidades de amostras agrupadas com relação aos horizontes bauxíticos estudados. Os agrupamentos podem ser visualizados no dendrograma apresentado na Figura $2 \mathrm{a}$. 
Tabela 1: Agrupamentos das amostras em cada horizonte bauxítico.

\begin{tabular}{ccccccccccc}
\hline \multirow{2}{*}{ Horizontes } & $\mathbf{1}$ & $\mathbf{2}$ & $\mathbf{3}$ & $\mathbf{4}$ & $\mathbf{5}$ & $\mathbf{6}$ & $\mathbf{7}$ & $\mathbf{8}$ & $\begin{array}{c}\text { Não } \\
\text { Agrupado }\end{array}$ & TOTAL \\
\hline$B N$ & - & - & - & - & - & 22 & 20 & - & - & 42 \\
$B N C$ & - & 12 & 7 & 1 & - & 1 & - & - & - & 21 \\
$L F$ & - & - & - & - & 16 & - & - & 10 & 1 & 27 \\
$B C$ & 1 & 3 & 22 & 21 & - & - & - & - & - & 47 \\
$B C B A$ & 3 & 8 & 20 & - & - & - & - & - & - & 31 \\
\hline TOTAL & 4 & 23 & 49 & 22 & 16 & 23 & 20 & 10 & 1 & 168 \\
\hline
\end{tabular}

O cluster 1 é um agrupamento outlier devido a discrepância da intensidade do pico referente ao plano $\mathrm{d}_{002}$ da gibbsita, o qual apresentou um número de contagem cerca de três vezes maior que o valor médio nas demais amostras (vide Anexo 1). Como pode-se observar esse é o grupo menos representativo e o mais dissemelhante. Um efeito de orientação preferencial associado à uma concentração maior de gibbsita nessas amostras pode ter causado esse resultado.

Conforme se observa na Figura 2a e na Tabela 1 os clusters 2 e 3 são os agrupamentos mais heterogêneos, englobando amostras dos horizontes BNC BC e BCBA. De acordo com os difratogramas de raios-X (Anexo 1), a característica comum entre esses grupos está na presença significativa de caulinita, "posição + intensidade" dos picos de Bragg $\mathrm{d}_{001} \mathrm{e} \mathrm{d}_{002}$. Em contrapartida, o fator dissemelhante entre eles é a intensidade do pico principal da gibbsita $\left(d_{002}\right)$, que nas amostras do cluster 3 é $~ 2 x$ maior que no cluster 2 .

Com relação aos clusters 2,3 e 4 observa-se uma tendência ao agrupamento das amostras ocasionado pelo aumento da gibbsita e diminuição continua da caulinita. Essa tendência é claramente visualizada na representação 3D do diagrama de componentes principais (Figura 2b). No cluster 4 são observadas, essencialmente, amostras do horizonte do minério principal (horizonte BC).

As amostras referentes ao horizonte laterita ferruginosa (LF) foram completamente individualizadas e, dessa forma, agrupadas exclusivamente nos clusters 5 e 8 . Por serem amostras ricas em minerais de ferro, tem-se, além das altas intensidades dos picos de Bragg das fases hematita e goethita, a alta intensidade do background devido ao efeito de fluorescência, característica essa que possibilita uma fácil individualização desse horizonte. A significativa dissemelhança observada no dendrograma da Figura 2a está relacionada a uma maior quantidade de gibbsita nas amostras do cluster 5, o que não ocorre naquelas agrupadas no cluster 8 . Tem-se ainda uma amostra LF que não foi agrupada por conter baixíssima quantidade de gibbsita.

De forma oposta, a mesma análise descrita anteriormente é observada entre os clusters 6 e 7, veja o diagrama de PCA na Figura 2b. Nota-se que a intensidade dos picos de Bragg da caulinita tendem a aumentar, e dos picos de gibbsita diminuir. Ambos os grupos são constituídos essencialmente de amostras do horizonte BN. 
(a)

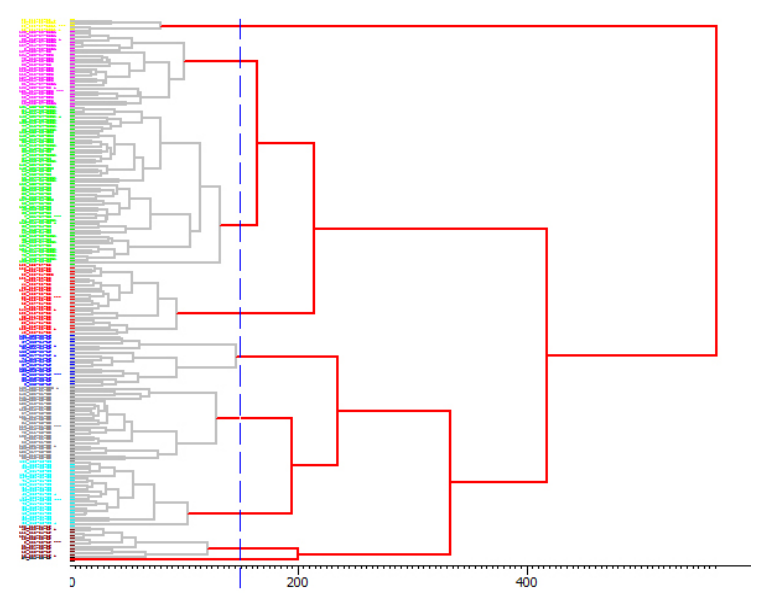

(b)

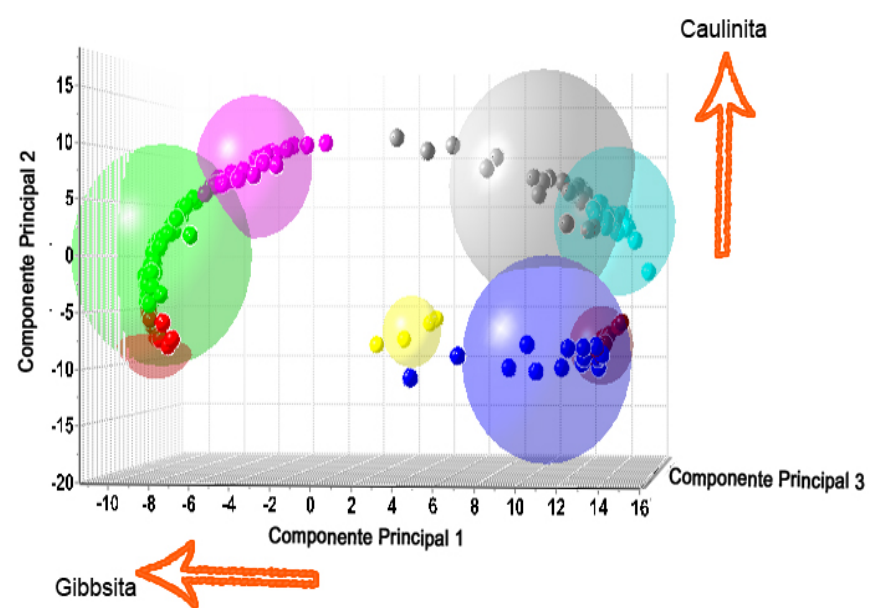

Legenda: cluster 1 = amarelo; cluster 2 = magenta; cluster 3 = verde; cluster 4 = vermelho; cluster $5=$ azul; cluster 6 = cinza; cluster 7 = ciano; cluster 8 = marrom; não agrupado = preto.

Figura 2: Discriminação dos agrupamentos: (a) Dendrograma e (b) Análise de Componentes Principais - PCA.

Atualmente, a classificação industrial das bauxitas é feita pela determinação indireta dos minerais: gibbsita e caulinita, através das medidas de Alumina Aproveitável $\left(\mathrm{AvAl}_{2} \mathrm{O}_{3}\right)$ e Sílica Reativa $\left(\mathrm{RxSiO}_{2}\right)$. Com base nos presentes resultados de análises de agrupamentos, pode-se estabelecer quais horizontes bauxíticos são economicamente viáveis para exploração, uma vez que para ser minério é preciso que as amostras apresentem elevada concentração do mineral de interesse (gibbsita $\leftrightarrow \mathrm{AvAl}_{2} \mathrm{O}_{3}$ ) e mínima do mineral deletério (caulinita $\leftrightarrow \mathrm{RxSiO}_{2}$ ) (Smith, 2009).

A Figura 3 apresenta a comparação entre os resultados da análise de agrupamentos e da análise química de $\mathrm{AvAl}_{2} \mathrm{O}_{3}$ e $\mathrm{RxSiO}$. Pode-se observar que há uma semelhança entre esse gráfico de dispersão e o diagrama de PCA da Figura $2 b$, onde se observa a mesma tendência descrita anteriormente, aumento e/ou diminuição das fases gibbsita e caulinita entre os grupos.

Observa-se que o agrupamento constituído essencialmente por amostras do horizonte BC (cluster 4) está estrito à uma pequena faixa de alto valores de $\mathrm{AvAl}_{2} \mathrm{O}_{3}(>47,5 \%)$ e baixos valores de $\mathrm{RxSiO}_{2}(<5 \%)$, que corresponde a faixa aceitável para minério.

Os grupos de amostras mais heterogêneos (clusters 3 e 4) que englobam os horizontes BC, $\mathrm{BCBA}$ e $\mathrm{BNC}$, embora apresentem valores de $\mathrm{AvAl}_{2} \mathrm{O}_{3}>47,5 \%$, apresentam também uma larga faixa de valores de $\mathrm{RxSiO}_{2}$ (1,38-7,38\%). Uma avaliação cuidadosa mostra que dentro desses grupos as amostras com maiores teores de $\mathrm{AvAl}_{2} \mathrm{O}_{3}$ e menores de $\mathrm{RxSiO}_{2}$ são justamente referentes ao horizonte do minério (BC), as amostras de $B N C$ apresentam valores intermediários e $\mathrm{BCBA}$ a maior variabilidade dos valores de $\mathrm{RxSiO}_{2}$. Dessa forma, industrialmente esses dois últimos horizontes são considerados estéreis ou minérios marginais durante a lavra (Almeida et al, 2012). 


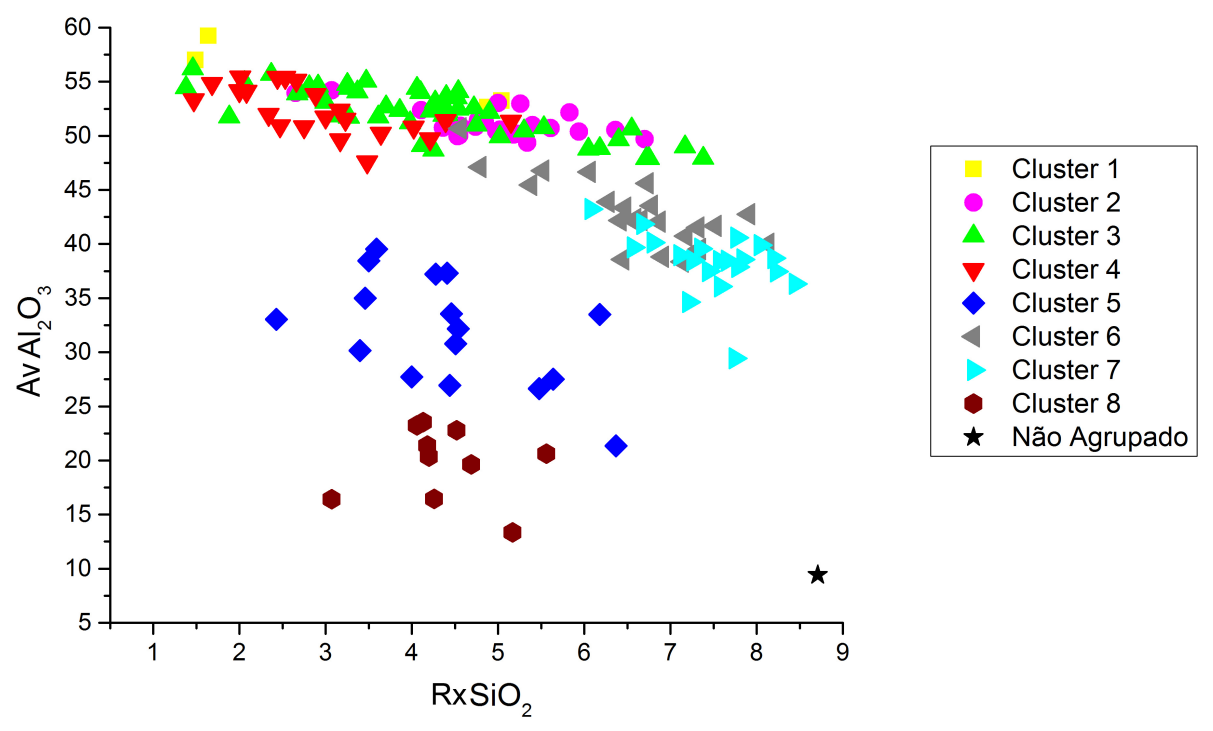

Figura 3: Gráfico de dispersão - Comparação entre análise de agrupamentos e valores de $\mathrm{AvAl}_{2} \mathrm{O}_{3}$ e $\mathrm{RxSiO}_{2}$.

Comumente, caulinitas apresentam diferenças significativas quanto ao seu grau de ordenamento estrutural, que podem ser ocasionadas por fatores geológicos, tais como: formação, transporte, deposição e tratamento mecânico. O baixo crescimento dos cristais e defeitos no empilhamento das folhas TOTOTO.... (T:tetraédrica e O:octaédrica) tornam caulinitas mais reativas aos processos hidrotérmicos (Cases et al., 1982, Ishida, 2010). Para se avaliar o grau de ordenamento estrutural das caulinitas presentes em cada horizonte foram selecionadas as amostras mais representativas de cada cluster e avaliado a largura à meia altura (FWHM - Full width at Half Maximum) dos picos referentes aos planos basais $\mathrm{d}_{001}$ e $\mathrm{d}_{002}$, semelhante a metodologia proposta por Amigó et al. (1994).

Os valores de FWHM na Figura 4 foram empilhados na mesma disposição do perfil litológico típico da região de Paragominas-PA. Esses valores estão correlacionados com os clusters obtidos na análise de agrupamentos. Observa-se uma tendência ao aumento de FWHM da base ao topo do perfil, mostrando que as caulinitas dos horizontes sobrejacentes são cada vez mais desordenadas. Essa variação é inerente a complexa gênese desses depósitos, relacionadas a diferentes ciclos de lateritização, com a formação de diferentes gerações de caulinita e de gibbsita (Costa, 1997). 


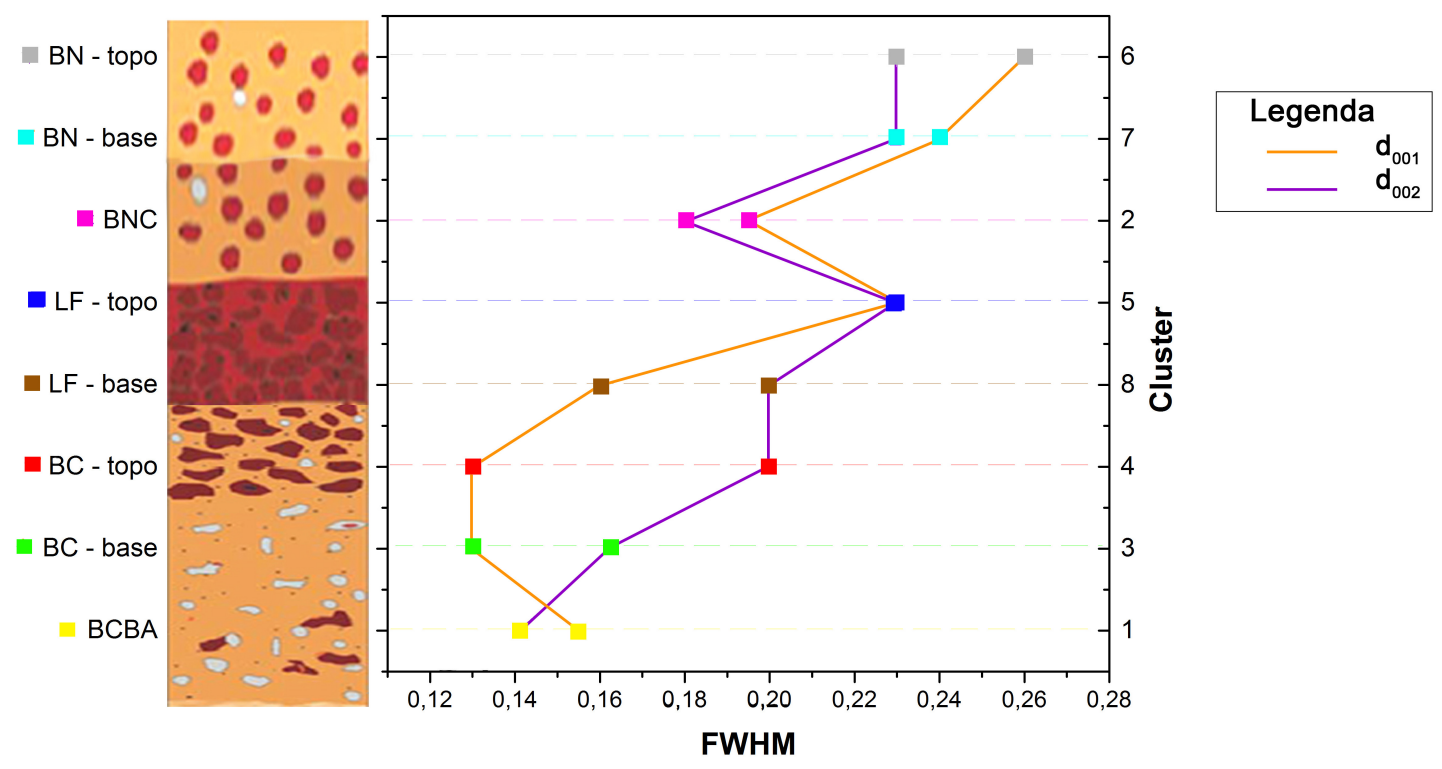

Figura 4: Comparação da medida de Largura à Meia Altura (FWHM) das amostras mais representativas de cada cluster - correlação com o perfil litológico típico da região de Paragominas-PA (perfil adaptado de Pereira, 2015).

\subsection{Classificação dos resíduos sólidos do processo de digestão Bayer}

Na digestão Bayer tanto a gibbsita quanto a caulinita são solubilizadas. No entanto, a sílica e alumina solúveis proveniente da caulinita reagem com hidróxido de sódio formando uma fase insolúvel, denominada sodalita Bayer (Melo et al., 2017). Por ser insolúvel e levar consigo parte do agente lixiviante $(\mathrm{NaOH})$, a formação de sodalita é indesejável no processo Bayer, o que exige um controle rigoroso do teor de $\mathrm{RxSiO}_{2}$ na bauxita.

As fases identificadas nos resíduos sólidos do processo de digestão Bayer foram aquelas insolúveis nas condições Bayer: goethita, hematita, anatásio e sodalita. Em algumas amostras foram identificadas caulinita e gibbsita remanescentes (difratogramas de raios-X no Anexo 2 ).

Os resíduos sólidos da digestão Bayer foram classificados em 5 grupos conforme apresentados na Figura 5. Observa-se que de forma geral, cada cluster agrupou as amostras dos mesmos horizontes (cluster $3=\mathrm{BN}$ e BNC, cluster $4=\mathrm{BC}$ e cluster $5=\mathrm{BCBA}$ ).

O tempo de reação de 60 min foi insuficiente para que toda caulinita fosse convertida em sodalita conforme observado na Figura 6, por est motivo os difratogramas destes residuos sólidos foram discriminados nos clusters 1 (BCBA e BC) e 2 (BNC e BN). No entanto, pela análise de agrupamentos (Figura 5) se observa uma grande dissimilaridade entre estes grupos, a qual constatou-se estar relacionada à intensidade dos picos de difração das fases caulinita e sodalita nestes materiais. Pela comparação dos difratogramas de raios- $X$ e análise da relação entre os picos principais destas duas fases ( $d_{001}$ e $d_{002}$ da caulinita e $d_{011}$ e $d_{112}$ da sodalita) foi observado que a intensidade dos picos da sodalita formada está relacionada à concentração inicial de caulinita na amostra, equanto a cristalinidade da sodalita (medida pela largura à meia altura) e a taxa de destruição da caulinita estão diretamente relacionados com a maior reatividade das caulinitas presentes em cada horizonte. Esta reatividade, por sua vez, está realacionada a disposição destes horizontes bauxíticos no perfil geológico conforme apresentado na Figura 4. Assim, as caulinitas de estrutura cristalina mais desordenada presente nos horizontes sobrejacentes (BNC e BN) são mais facilmente lixiviadas e convertidas em sodalita Bayer. 
Em sequência a este estudo Melo (2016) investigou a influênica da reatividade da caulinita dessas amostras na composição química e estrutura cristalina das fases formadas. Uma discussão cristaloquímica mais detalhada sobre isso pode ser consultada em Melo et al. (2017).

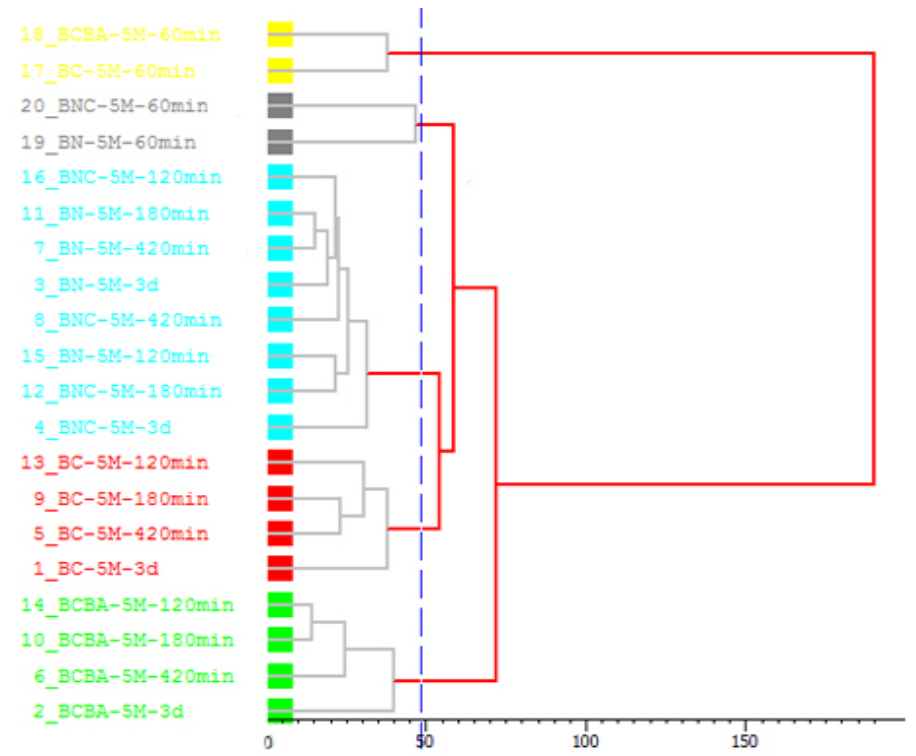

Figura 5: Dendrograma dos resíduos sólidos da digestão Bayer.

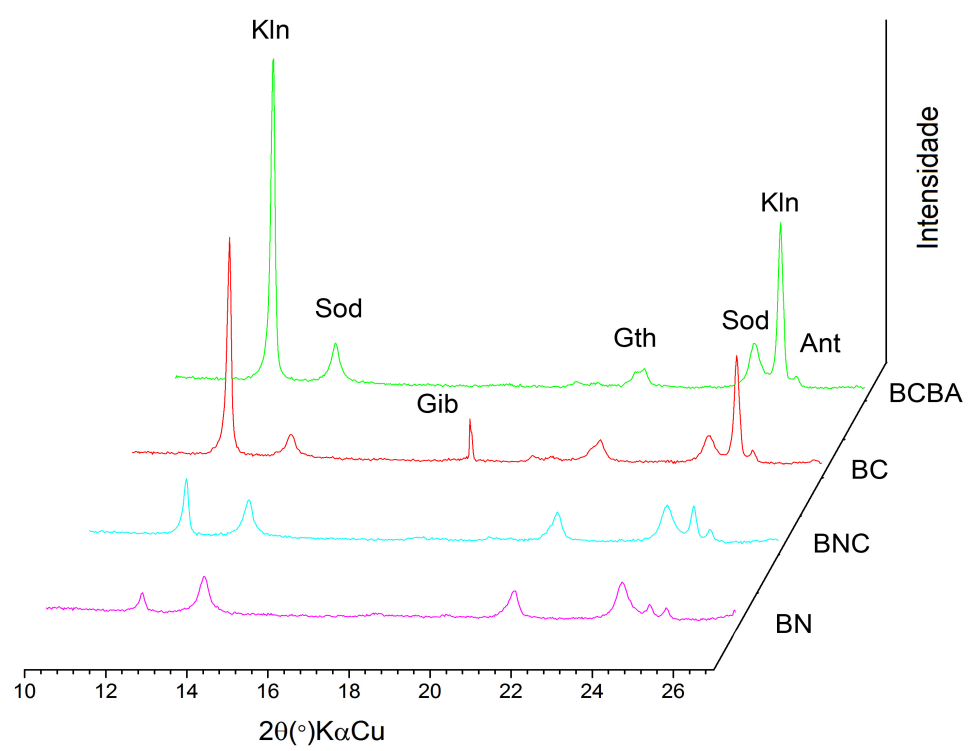

Legenda: KIn: Caulinita; Sod: Sodalita Bayer; Gib: Gibbsita; Gth: Goethita; Ant: Anatásio.

Figura 6: Comparação dos difratogramas de raios- $X$ dos resíduos sólidos da digestão Bayer (60 min).

\section{CONCLUSÕES}

A análise de agrupamentos associada a difratometria de raios- $X$ se mostrou uma ferramenta prática e útil na demarcação dos horizontes bauxíticos de acordo com suas características cristaloquímicas. Assim, pôde-se classificar as amostras de maneira satisfatória: tanto em relação ao teor dos minerais de interesse e deletério, gibbsita e caulinita, 
respectivamente; quanto levando em consideração o grau de ordenamento estrutural e a reatividade das caulinitas presentes.

Com relação aos resíduos sólidos da digestão Bayer, a análise de agrupamentos também permitiu demarcar os horizontes bauxíticos, além de revelar quais caulinitas eram mais reativas.

\section{AGRADECIMENTOS}

Os autores agradecem a Hydro Mineração Paragominas pelas amostras e análises cedidas, e CAPES pelas bolsas de mestrado e doutorado do primeiro autor, de mestrado do segundo autor. Ao CNPq Edital MCT/CT-Mineral/VALE/CNPq no 12/2009, 550.297/2010-3); e a FAPESPA Edital 01/2010, ICAAF no 027/2011, pelo apoio financeiro.

\section{REFERÊNCIAS}

Almeida, D. S. G., Silva, F. A. N. G., Santos, C. G. M., Medeiros, M. E., Sampaio, J. A., Garrido, F. M. S. (2012). Estudo de beneficiamento químico e físico e caracterização de bauxitas do Pará. HOLOS. 5, 115-124.

Amigó, J. M., Bastida, J., Sanz, A., Signes, M., Serrano, J Crystallinity of lower cretaceous kaolinities of Teruel - Spain. Applied Clay Science. 9, 51-69.

Cases, J. M., Liétard, O., Yvon, J., Delon, J. F. (1982). Etude des proprietes cristallochimiques, morphologiques, superficielles, de kaolinites désordonnés. Bull. Mineral. 105, 439-455.

Costa, M. L. (1997). Lateritization as a major process of ore deposit formation in the Amazon region. Exploration and Mining Geology. 6, 79-104.

Freyssinet, Ph., Butt, C. R. M., Morris, R. C., Piantone, P. (2005). Ore-forming process related to lateritic weathering. Economic Geology. 100, 681-722.

Ishida, D. A. (2010). Caracterização e gênese de solos e de depósitos de caulim associado, São Gabriel da Cachoeira - AM. Tese de Doutorado, Instituto de Geociências, Universidade de São Paulo, 172.

Kotschoubey, B., Truckenbrodt, W., Calaf, J. M. C. (2005). Evolução geológica da porção meridional da província bauxitífera de Paragomias durante o Neógeno/Pleistoceno (nordeste da Bacia do Grajaú, nordeste do Pará e extremo oeste de Maranhão). Rev. Bras. Geoc. 35(2), 263-272.

Macchiarola, K., Gobbo, L., Campbel. I., Mcdonald. A. M., Cirelli, J. (2007). Modern X-Ray diffraction techniques for exploration and analysis of ore bodies. In: Proceedings of Exploration 07: Fifth Decennial International Conference on Mineral Exploration. Geochemical Case Histories \& Geochemical Exploration Methods. 86, 1007-1011.

Melo, C. C. A. (2016). Cristaloquímica da sodalita Bayer derivada de bauxitas com alta sílica reativa de Paragominas-PA. Dissertação de Mestrado. Instituto de Geociências, Universidade Federal do Pará.

Melo, C. C. A., Paz, S. P. A., Angélica, R. S. (2017). Fases sodalita formada a partir de caulinitas amazônicas sob condições de digestão Bayer. Matéria (Rio de Janeiro), 22(3), e11862.

Paz, S. P. A., Angélica, R. S., Kahn, H. (2017a). Optimization of the reactive silica quantification 
method applied to Paragominas-type gibbsitic bauxites. International Journal of Mineral Processing. 162, 48-57.

Paz, S. P. A., Torres, P. W. T. S., Angélica, R. S., Kahn, H. (2017b). Synthesis, Rietveld refinement and DSC analysis of Al-goethites to support mineralogical quantification of gibbsitic bauxites. Journal of Thermal Analysis and Calorimetry. 128, 841-854.

Peixoto, S. F. \& Horbe, A. M. C. (2008). Bauxitas do nordeste do Amazonas. Rev. Bras. Geoc. $38(2), 406-422$.

Pereira, C. B. (2015). Mineralogia e geoquímica dos perfis bauxíticos da mina Miltônia 3, região de Paragominas-PA. Dissertação de Mestrado. Instituto de Geociências, Universidade Federal do Pará.

Shimizu, V. K., Kahn, H., Antoniassi, J. L., Ulsen, C. (2012). Copper ore type definition from Sossego Mine using X-ray diffraction and cluster analysis technique. REM: Rev. Esc. Minas. 65(4), 561-566.

Silva, H. M., Picanço, E., Maurity, C., Morais, W., Santos, H. C., Guimarães, O. (2008). Geology, mining operation and scheduling of the Paragominas bauxite mine. Proceedings of the 8th International Alumina Quality Workshop, p. 11-16.

Smith, P. (2009). The processing of high silica bauxites - Review of existing and potential processes. Hidrometallurgy. 98, 162-176.

Thiel, R. (1963). Zum system $\alpha-\mathrm{FeOOH}-\alpha-\mathrm{AlOOH}$. Anorganische und Allgemeine Chemie. 329, 7078. 